\title{
Kelvin-Helmholtz vortices and secondary instabilities in super-magnetosonic regimes
}

\author{
F. Palermo, M. Faganello, F. Califano, and F. Pegoraro \\ Dip. Fisica, Università di Pisa and CNISM, Pisa, Italy \\ Received: 28 February 2011 - Revised: 23 May 2011 - Accepted: 14 June 2011 - Published: 29 June 2011
}

\begin{abstract}
The nonlinear behaviour of the Kelvin-Helmholtz instability is investigated with a two-fluid simulation code in both sub-magnetosonic and super-magnetosonic regimes in a two-dimensional configuration chosen so as to represent typical conditions observed at the Earth's magnetopause flanks. It is shown that in super-magnetosonic regimes the plasma density inside the vortices produced by the development of the Kelvin-Helmholtz instability is approximately uniform, making the plasma inside the vortices effectively stable against the onset of secondary instabilities. However, the relative motion of the vortices relative to the plasma flow can cause the formation of shock structures. It is shown that in the region where the shocks are attached to the vortex boundaries the plasma conditions change rapidly and develop large gradients that allow for the onset of secondary instabilities not observed in sub-magnetosonic regimes.
\end{abstract}

Keywords. Magnetospheric physics (Solar windmagnetosphere interactions)

\section{Introduction}

The solar wind and the Earth's magnetosphere interact through a range of different physical processes in different regions around the magnetopause. The effect of these processes and the competition between them depend on the specific plasma conditions and understanding them is a fundamental problem of magnetospheric physics. In particular the interplanetary magnetic field (IMF) and its orientation with respect to the geomagnetic field (GMF) play a very important role. When the IMF orientation is nearly southwards magnetic reconnection is expected to be the main mechanism through which plasma and energy from the magnetized

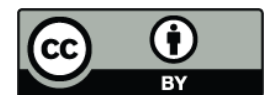

Correspondence to: F. Palermo (palermo@df.unipi.it) solar wind enter the magnetosphere. This mechanism acts mainly at locations where the IMF is antiparallel to the direction of the GMF, i.e. at low Earth latitudes. The occurrence of magnetic reconnection and its related plasma and energy transport have been observed by probes and discussed in the literature, (see, e.g., Phan et al., 2000; Nishida et al., 1996).

On the contrary, the reconnection process is strongly reduced when the IMF is parallel to the GMF. In this case we would not expect a large transport of plasma and energy or, in general, a strong interaction between the solar wind and the magnetosphere. However, during the period in which the IMF points northwards, a strong mixing layer is observed (Lennartsson and Shelley, 1986; Mitchell et al., 1987; Fujimoto et al., 1998) at low latitudes. In order to account for this observed exchange of mass and energy it was proposed (Belmont and Chanteur, 1989; Otto and Fairfield, 2000; Nakamura and Fujimoto, 2005) that the velocity shear between the magnetosphere and the magnetosheath leads to the onset of the Kelvin-Helmholtz (KH) instability. As discussed in Miura $(1984,1987)$, the nonlinear development of the KH instability can generate an anomalous viscosity through the formation of vortex structures at the magnetopause flanks. This analysis provides a reformulation of one of the early models (Axford and Hines, 1961) in which the solar wind momentum can enter the magnetosphere through diffusive viscous processes. Evidence of the KH instability at the low latitude magnetopause flanks has been reported in several articles (Hasegawa et al., 2004; Fairfield et al., 2000). Unlike reconnection, this process takes place independently of whether the solar wind magnetic field is parallel or antiparallel to the field in the magnetosphere, but only if its component parallel to the flow is sufficiently small in order that magnetic tension does not suppress the KH instability. In addition the onset of this instability and its development depend on the plasma conditions such as its density and velocity shear, thus involving a large variety of regimes and configurations, several aspects of which are not yet fully understood.

Published by Copernicus Publications on behalf of the European Geosciences Union. 
A detailed analysis (Fairfield et al., 2000) of Geotail data on an event on 24 March 1995 when the interplanetary magnetic field was pointing northwards showed clear evidence of signatures of $\mathrm{KH}$ vortices. Moreover, by simulating this event in the framework of a 2-D MHD plasma description, this study showed that the KH instability can twist the field lines of the magnetic field leading to the onset of magnetic reconnection inside the vortices (Otto and Fairfield, 2000). In these simulations the authors considered an initial configuration where the magnetic field is in the same direction in the magnetosheath and in the magnetosphere regions, thus excluding the occurrence of reconnection in the plasma initial configuration. They concluded that the $\mathrm{KH}$ instability allows not only for the entry of energy and momentum into the magnetosphere at the equatorial flanks, but also for the entry of mass from the magnetosheath plasma.

Later Nykyri and Otto (2001) quantified the effect of the reconnection process inside the $\mathrm{KH}$ vortices by means of 2-D MHD simulations showing that reconnection can be an important mechanism that brings plasma from the solar wind into the magnetosphere. Moreover, they showed that the onset of secondary reconnection instabilities can disrupt the vortex structure and change the large scale evolution of the configuration significantly. In the low latitude magnetopause context, the Rayleigh Taylor (RT) instability (Matsumoto and Hoshino, 2004; Faganello et al., 2008a) is another important secondary instability that can develop inside the $\mathrm{KH}$ vortices. This instability is driven by the alternating density layers consisting of solar wind and of magnetosphere plasma respectively which are rolled up in the KH vortex arms. If the initial density difference is large enough, the vortex centrifugal acceleration, acting as an "effective gravity", makes the vortex arms RT unstable. During the nonlinear evolution of the vortices, secondary fluid instabilities (RT but also $\mathrm{KH}$ ) compete with the vortex pairing which is expected to occur in a KH vortex chain system (Faganello et al., 2008a). The onset of the RT instability can interrupt the vortex pairing process and can eventually lead to the generation of a turbulent mixing layer. Furthermore, magnetic reconnection can occur inside and between the vortices and change the global magnetic topology as well as the large scale vortex evolution in the region between the solar wind and the magnetosphere (Nakamura and Fujimoto, 2005; Faganello et al., 2008b,c; Liu and Hu, 1988). Recently, it has been shown that the competition between vortex pairing and secondary $\mathrm{KH}$ and RT instabilities and magnetic reconnection in typical magnetospheric conditions leads to the formation of large scale vortex structures filled by magnetic islands. These islands are generated by a RT induced reconnection process (Tenerani et al., 2010) with a typical width of the order of the ion skin depth.

Most of these investigations have considered a submagnetosonic flow in the magnetosheath region, thus a priori excluding the possibility of generating shock structures induced by the presence of vortices in the flow. Indeed, the physical properties of the solar wind change when crossing the Earth's bow shock and moving tailward inside the magnetosheath. In fact, crossing the bow shock, the supersonic and super-Alfvénic solar wind velocity, referred to the Earth obstacle, is reduced and, according to the Rankine-Hugoniot relationships, the plasma density and temperature increase thus leading to subsonic velocities. However, at larger distances, the shocked solar wind regains a large fraction of its initial speed as it flows past the magnetosphere and, at the same time, the plasma temperature progressively decreases making the solar plasma supersonic, as explained in Spreiter et al. (1966) by means of a gasdynamical model. In this model the solar wind becomes supersonic in the magnetosheath, but its flow velocity is always smaller than that of the flow outside the magnetosheath. By including the presence of a magnetic field in a configuration in which the interplanetary magnetic field is parallel to geomagnetic field lines (a condition in which reconnection is weak) in Chen et al. (1993) magnetic tension was shown to play an important role in accelerating the plasma in the magnetosheath. In this article, which was supported by observational data, the authors showed that the plasma velocity in the magnetosheath can be larger than the solar wind velocity outside the magnetosheath. These results indicate that super-magnetosonic regimes should play an important role in the development of the KH instability under magnetopause and magnetotail conditions.

The most important feature concerning the transition towards magnetosonic Mach numbers of the order of (or larger than) unity, is the role of the vortices that act as obstacles and can lead to the formation of shock structures. These structures can extend far from the transition region between the magnetosphere and the magnetosheath, similar to what found in the ideal MHD limit (Miura, 1982, 1990, 1992) and in the purely hydrodynamic limit (Kobayashi et al., 2008). Furthermore, during the shock formation, strong rarefaction and compressional effects occur in the transition region and modify the non linear processes (pairing, onset of secondary instabilities) observed in the low magnetosonic Mach number regime. In a supersonic regime the free energy stored in the equilibrium configuration is available both for the secondary phenomena that act during the nonlinear evolution of $\mathrm{KH}$ instability as well as for the formation of shocks induced by the presence of vortices.

In a recent article (Palermo et al., 2011) we have shown that the development of shocks in correspondence of vortices is associated to vortex rarefaction effects that tend to stabilize the inner vortex region against secondary instabilities. However, on longer times, secondary instabilities can develop along the outer vortex boundary. Due to computational reasons and to the fact that we focused on the problem of shock formation, the long time development was not considered in Palermo et al. (2011). In the present article, we address the study of the long time evolution where, after the shock formation, secondary instabilities develop in an initial supersonic regime. In particular, we show that these 
instabilities form at special locations near the vortex boundaries downstream from the shocks. Then, we discuss the mechanisms that can be responsible for the onset of these instabilities. We stress that secondary instabilities have been previously studied only in the sub-magnetosonic regime.

In the following we fix the initial magnetic field angle with respect to the plane of the flow velocity and vary the intensity of the sheared velocity field and the density profile in order to study the nonlinear evolution of the $\mathrm{KH}$ instability in super-magnetosonic regimes with typical Mach numbers of order one or larger. Here the magnetic field is in the same direction in the magnetosphere and in the magnetosheath but anti-parallel magnetic layers in the flow velocity plane are generated by the KH vortex motion. We stress that the supersonic and/or super-magnetosonic regimes are in general defined with respect to the solar wind velocity, while here we are mostly interested in supersonic and/or supermagnetosonic Mach numbers calculated by considering the velocity difference between the $\mathrm{KH}$ vortices and the large scale flow (Miura, 1990, 1992).

The present article is organized as follows. In Sect. 2 we briefly recall the set of equations that are integrated in our simulations and the numerical method adopted. In Sect. 3, after recalling the main results discussed in Palermo et al. (2011), we present the results of the new simulation runs emphasizing that, although the vortex configuration is globally more stable than in a sub-magnetosonic regime, the physical plasma conditions vary very rapidly in the region at the vortex border near the shock leading to the development of a local secondary instabilities.

We begin by describing the $\mathrm{KH}$ instability in a supermagnetosonic regime with large values of the velocity and density jumps between the magnetosphere and the magnetosheath. However, in this case we do not observe the "standard" development of the KH instability into fully fledged secondary vortex structures, and the secondary instabilities are limited to the region around the external boundary of the vortex. Then, we decrease the velocity and density jumps which tends to stabilize the vortex structure by reducing the growth of the instabilities observed at the outer boundaries. Nevertheless, at later time secondary instabilities do develop driven by the pairing process that stretch and compress the fluid thus locally creating the conditions for the secondary instabilities to develop. Finally, we reduce the velocity jump further and keep now an uniform density and temperature distribution. This allows us to investigate the competition between the vortex pairing, the onset of secondary KH and reconnection instabilities and the generation of shocks under conditions that extend those investigated in Faganello et al. (2008c).

\section{Numerical method}

Following the approach adopted in our previous investigation (see Palermo et al., 2011) of the nonlinear development of the KH instability in super-magnetosonic regimes we use a two-fluid description of the plasma dynamics (see Faganello et al., 2009, for details). The equations for the electron and ion fluids (indicated by the subscripts "e", "i", respectively) are set in dimensionless form with time normalized to the inverse of the ion gyrofrequency, space to the ion inertial scale length $d_{\mathrm{i}}=c / \omega_{\mathrm{pi}}$ (with $\omega_{\mathrm{i}}$, the ion plasma frequency and $c$ the speed of light), and consequently velocities to the Alfvén velocity $c_{\mathrm{A}}$

$$
\begin{aligned}
& \frac{\partial n}{\partial t}+\nabla \cdot(n \boldsymbol{U})=0, \\
& \frac{\partial(n \boldsymbol{U})}{\partial t}+\nabla \cdot\left[n\left(\boldsymbol{u}_{\mathrm{i}} \boldsymbol{u}_{\mathrm{i}}+d_{\mathrm{e}}^{2} \boldsymbol{u}_{\mathrm{e}} \boldsymbol{u}_{\mathrm{e}}\right)+P \overline{\overline{\mathbf{I}}}-\boldsymbol{B} \boldsymbol{B}\right]=0,
\end{aligned}
$$

where $\boldsymbol{U}=\boldsymbol{u}_{\mathrm{i}}+d_{\mathrm{e}}^{2} \boldsymbol{u}_{\mathrm{e}}$ is the fluid velocity, $P=P_{\mathrm{i}}+P_{\mathrm{e}}$ the pressure and $n=n_{i} \approx n_{e}$ the density (quasi-neutrality is assumed). We adopt an adiabatic closure equation

$$
\frac{\partial\left(n S_{\mathrm{e}, \mathrm{i}}\right)}{\partial t}+\nabla \cdot\left(n S_{\mathrm{e}, \mathrm{i}} \boldsymbol{u}_{\mathrm{e}, \mathrm{i}}\right)=0,
$$

where $S_{\mathrm{e}, \mathrm{i}}=P_{\mathrm{e}, \mathrm{i}} n^{-\gamma}$ and $\gamma=5 / 3$. The dimensionless sound velocity is defined as $c_{\mathrm{S}}=(\gamma P / n)^{1 / 2}$. The electric field $\boldsymbol{E}$ is calculated by means of a generalized Ohm's law (Valentini et al., 2007) that includes electron inertia effects

$$
\begin{aligned}
\left(1-d_{\mathrm{e}}^{2} \nabla^{2}\right) \boldsymbol{E}= & -\boldsymbol{u}_{\mathrm{e}} \times \boldsymbol{B}-\frac{1}{n} \nabla P_{\mathrm{e}} \\
& +d_{\mathrm{e}}^{2}\left\{-\boldsymbol{u}_{\mathrm{i}} \times \boldsymbol{B}+\frac{1}{n} \nabla \cdot\left[n\left(\boldsymbol{u}_{\mathrm{i}} \boldsymbol{u}_{\mathrm{i}}-\boldsymbol{u}_{\mathrm{e}} \boldsymbol{u}_{\mathrm{e}}\right)\right]\right\},
\end{aligned}
$$

and the magnetic field is calculated by solving Faraday's equation

$$
\partial \boldsymbol{B} / \partial t=-\nabla \times \boldsymbol{E} .
$$

We consider a 2-D, $L_{\mathrm{x}} \times L_{\mathrm{y}}$, spatial domain with 3 -D velocity and electromagnetic fields. The initial equilibrium configuration is taken to be inhomogeneous along the $\mathrm{x}$ direction. Periodic boundary conditions are imposed along the $y$-direction corresponding to the solar wind direction. We take open boundary conditions in the inhomogeneous $\mathrm{x}$ direction based on the MHD characteristic decomposition. In this way, all the perturbations generated in the central region of the simulation box where the $\mathrm{KH}$ vortices develop leave the numerical domain without reflection. The idea is that, except for the zone where the $\mathrm{KH}$ vortices develop and where a separate electron and ion dynamics is possible, far from the central region the plasma dynamics remains "slow" so that the MHD approximation holds and MHD boundary conditions can be used. These transparent conditions are obtained from the ideal MHD set of hyperbolic equations for 
Table 1. The values of the quantities for the different simulation cases.

\begin{tabular}{lllllll}
\hline & $\Delta n$ & $\Delta T_{\mathrm{i}}$ & $\Delta T_{\mathrm{e}}$ & $T_{0, \mathrm{i}}$ & $T_{0, \mathrm{e}}$ & $\theta$ \\
\hline Case $V_{0}=6$ & 0.9 & 2.4 & 0.4 & 0.6 & 0.4 & 0.02 \\
Case $V_{0}=4.6$ & 0.7 & 0.6 & 0.4 & 0.6 & 0.4 & 0.02 \\
Case $V_{0}=2$ & 0 & 0 & 0 & 0.5 & 0.5 & 0.05 \\
\hline
\end{tabular}

which it is possible to define projected characteristic along the x-direction. For a detailed derivation of these boundary conditions see Faganello et al. (2009).

Numerical stability is achieved by means of filters, a spectral filter along the periodic y-direction and a sixth-order spectral-like filtering scheme along the inhomogeneous $\mathrm{x}$ direction (Lele, 1992). In the simulations the left side of the computational box, $x<0$, is taken to represent the magnetospheric region and the right hand side, $x>0$, the magnetosheath region with a transition layer around $x=0$.

We choose a reference frame where the magnetospheric and the magnetosheath plasma velocities are initially in the $\mathrm{y}$-direction and are equal and opposite

$\boldsymbol{V}_{\mathrm{eq}}=\frac{V_{0}}{2} \tanh \left(x / L_{\mathrm{eq}}\right) \boldsymbol{e}_{\mathrm{y}}$.

The initial density and temperature profiles are chosen as

$n(x)=n_{0}-\frac{\Delta n}{2}\left[\left(1-\tanh \left(x / L_{\mathrm{eq}}\right)\right]\right.$,

$T_{\mathrm{e}, \mathrm{i}}(x)=T_{0 ; \mathrm{e}, \mathrm{i}}+\frac{\Delta T_{\mathrm{e}, \mathrm{i}}}{2}\left[\left(1-\tanh \left(x / L_{\mathrm{eq}}\right)\right]\right.$,

where $\Delta n, \Delta T_{\mathrm{e}}$ and $\Delta T_{\mathrm{i}}$ are the values of the density, electron and ion temperature jump between the magnetosheath $(R)$ and the magnetosphere $(L)$ plasma, with $T_{0, \mathrm{e}}+T_{0, \mathrm{i}}=1$ and $n_{0}=1$.

The values of the quantities $\Delta n, \Delta T_{\mathrm{i}}, \Delta T_{\mathrm{e}}, T_{0, \mathrm{i}}, T_{0, \mathrm{e}}$ are specified in Table 1 and, for the sake of clarity, in the following section for each simulation. The different simulation cases are identified by the value of $V_{0}$.

All quantities are normalized using the characteristic values at the magnetosheath side (the outermost region). The plasma density increases while the temperature decreases from the magnetosphere to the magnetosheath, as consistent with satellite observations. For the sake of simplicity we have assumed that all equilibrium quantities vary on the same scale length $L_{\mathrm{eq}}$ (we take $L_{\mathrm{eq}}=3$ corresponding to a transition layer wider than the ion inertial length). The magnetic field $B_{0}(x)$ is chosen such that the sum of the thermal pressure and the magnetic pressure is initially uniform in the $x$ (inhomogeneous) direction

$B_{0}(x)=\left[B_{0, R}^{2}+2\left(P_{0, R}-P_{0}(x)\right)\right]^{1 / 2}$,

with components $B_{\mathrm{y}}(x)=B_{0}(x) \sin \theta$ and $B_{\mathrm{z}}(x)=$ $B_{0}(x) \cos \theta$. For each discussed case we choose a $\theta$ value to make the magnetic field almost perpendicular to the $(\mathrm{x}, \mathrm{y})$-plane, in agreement with the low latitude magnetosphere-magnetosheath system during northwards magnetic field configurations when the solar wind and the geomagnetic field lines are parallel (see Table 1).

We define the solar wind fast magnetosonic Mach number in the Earth rest frame as

$M_{f}^{\mathrm{sw}}=V^{\mathrm{sw}} / c_{\mathrm{f}} ; \quad c_{\mathrm{f}}=\left(c_{\mathrm{s}}^{2}+c_{\mathrm{A}}^{2}\right)^{1 / 2}$,

where $c_{\mathrm{s}}$ and $c_{\mathrm{A}}$ are the sound and Alfvén velocity, $c_{\mathrm{f}}$ is the fast magnetosonic velocity calculated at the magnetosheath boundary and $V^{\mathrm{sw}}=V_{0}$ is the total velocity jump.

For numerical reasons the mass ratio is fixed as $m_{\mathrm{i}} / m_{\mathrm{e}}=64$.

We take a simulation box with $L_{\mathrm{x}}=120$ and $L_{\mathrm{y}}=60 \pi$ and a grid mesh $d x \simeq d y \simeq 0.1$ in units of $d_{\mathrm{i}}$. This simulation box is large enough compared to the typical size of the vortex structures expected after the development of the $\mathrm{KH}$ instability in the central region of the simulation box.

\section{Simulation results}

Before discussing the new results obtained with our two fluid simulations regarding the onset of secondary $\mathrm{KH}$ instabilities in a super-magnetosonic regime, we will summarize briefly the main points recently addressed in Palermo et al. (2011). In this paper the transition between the submagnetosonic and the super-magnetosonic regime was investigated by varying the solar wind velocity, as controlled by the parameter $V_{0}$ in Eq. (6). It was shown in particular that the value of the density jump plays a crucial role in the development of the nonlinear phase of the KH instability especially at super-magnetosonic Mach numbers.

We recall that in the case of a uniform density plasma the $\mathrm{KH}$ vortices would remain at rest in the reference frame where the flow velocity in the asymptotic magnetosheath and magnetospheric regions takes the values $\pm V_{0} / 2$ and the plasma is at rest at the center, $x=0$, see Eq. (6). On the contrary, in the presence of a density gradient, the $\mathrm{KH}$ vortices propagate in the direction of the flow of the denser plasma (in our case the magnetosheath region in the right hand side of the simulation box). For an incompressible plasma with a discontinuity in the density and velocity profiles $\left(L_{\mathrm{eq}} \rightarrow 0\right.$ in Eqs. (6), (7), the vortex velocity can be estimated as (Otto and Fairfield, 2000)

$V_{\text {theor }}^{\text {vort }}=\frac{V_{0}}{2}\left(n_{0, R}-n_{0, L}\right) /\left(n_{0, R}+n_{0, L}\right)$.

Since the KH vortices act as "obstacles" in the fluid flow, determining the propagation vortex velocity induced by the density variation is of crucial importance for the interpretation of the shock generation.

For this purpose, as in Palermo et al. (2011), we define the vortex Mach number in our simulation frame analogously 
to the "convective Mach number" defined in Miura (1990, 1992), as

$M_{\mathrm{f}, L / R}^{\text {vort }}=U_{L / R} / c_{\mathrm{f}, L / R}, \quad U_{L / R}=\left\|V_{0} / 2 \mp V_{\text {vort }}\right\|$,

where $U$ is the relative velocity of the vortex with respect to the magnetospheric $(L)$ or to the magnetosheath $(R)$ flow, $c_{\mathrm{f}}$ is the fast magnetosonic velocity in the magnetospheric $(L)$ or in the magnetosheath $(R)$ side, while $V_{\text {vort }}$ is extrapolated from the simulations. As explained in Palermo et al. (2011) compressibility effects become increasingly important for larger values of the flow velocity and the value of the vortex velocity $V_{\text {vort }}$ is found to be lower than that predicted by Eq. (11). Therefore, Mach numbers $M_{\mathrm{f} R}^{\mathrm{vort}} \gtrsim 1$ in the magnetosheath are obtained for values of the solar wind velocity $V_{0}$ lower than that predicted on the basis of Eq. (12). At Mach number $M_{\mathrm{f} R}^{\text {vort }} \gtrsim 1$ vortices are seen as super-magnetosonic obstacles by the flow and thus in correspondence to each vortex a shock structure develops outwards in the magnetosheath region. In this regime the physical conditions inside the vortices make them essentially stable against the development of secondary instabilities. As mentioned in the introduction, in the present paper we show that secondary instabilities can however develop downstream from the shocks near the vortex boundaries and discuss the mechanisms that can allow for the onset of these instabilities. Before discussing the results obtained in the new simulations, we recall that since the magnetospheric plasma density is lower, the fast Mach number is larger on the magnetosheath side where we thus find more favourable conditions for shock formation.

First we present the case with $V_{0}=6$. For this case we take $\Delta n=0.9$ which corresponds to a factor ten for the density jump in Eq. (7) between the magnetosphere and the magnetosheath. This is a value typically observed at the magnetopause flanks. In addition we choose $\Delta T_{\mathrm{i}}=2.4$, $\Delta T_{\mathrm{e}}=0.4, \quad T_{0, \mathrm{i}}=0.6, \quad T_{0, \mathrm{e}}=0.4$ in Eq. (8) and we consider an angle $\theta=0.02$. The typical vortex speed observed in the simulations in this case is $V_{\text {vort }} \sim 1.2$ that turns out to be significantly smaller than the value $V_{\text {theor }}^{\text {vort }} \simeq 2.4$ expected from Eq. (11). This corresponds to $M_{\mathrm{f}, R}^{\text {vort }}=1.1$ (instead of $M_{\mathrm{f}, R}^{\text {vort }}=0.4$ as would be the case for $\left.V_{\text {theor }}^{\text {vort }}\right)$ and consequently we observe the formation of magnetosonic shocks in the magnetosheath region. This is shown in Fig. 1 where at $t=160$ we observe three well developed vortices and, in correspondence to each vortex, a shock structure that extends outward into the magnetosheath region.

On the magnetosphere side we have a $M_{\mathrm{f}, L}^{\text {vort }}=0.8$, however the velocity enhancement (Miura, 1984) near the vortex in correspondence of $y \sim 140$ allows us to obtain a local Mach number larger than one and we thus observe a (weaker) shock structure that extends into the magnetosphere.

A common feature that emerges from the study of the development of the $\mathrm{KH}$ vortices in super-magnetosonic regimes is that, unlike the sub-magnetosonic case, vortices appear to consist of nearly uniform, low density plasma (see, e.g.,

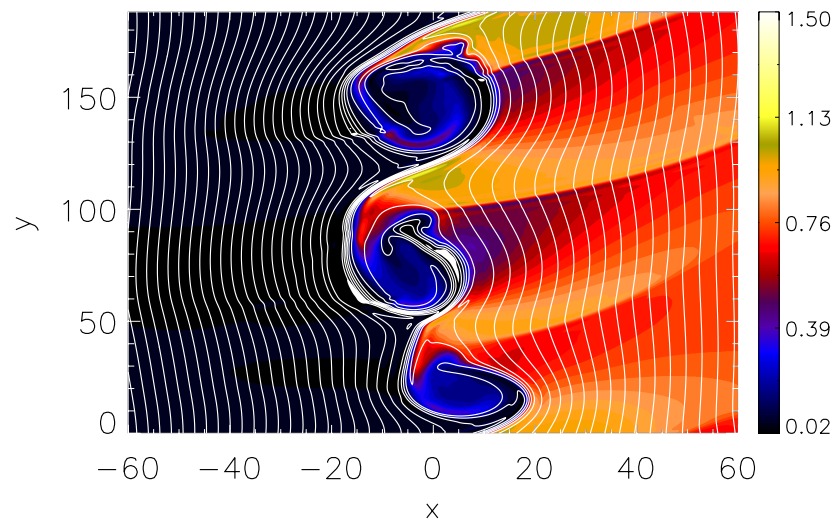

Fig. 1. Shaded density isocontours at $t=160, V_{0}=6$. White curves represent magnetic field lines in the (x,y)-plane.

Fig. 1). In the sub-magnetosonic case, instead, the vortices are composed of spiralling arms of alternating right-side and left-side values of the density, as discussed in Faganello et al. (2008a) and in Tenerani et al. (2010) in configurations with and, respectively, without an in-plane magnetic field component. The characteristic value of the vortex density is of the order of the magnetospheric density. Since the density inside these vortices is approximately uniform, the vortex structures can be expected to be stable with respect to secondary instabilities. However, at the vortex boundaries the local values of the velocity shear and of the density jump are still large enough to excite small scale instabilities (see for example Fig. 1 at $x \sim 10, y \sim 160$ ). As a result, the external boundary of the vortices starts to bend and/or generate local bumps. This is shown in Fig. 2 where we draw the isocontours of the density at $t=156$ for the top vortex centered at $x \sim 0, y \sim 140$. We observe the initial development of local instabilities at the boundaries, evidenced by the bending of the magnetic field lines downstream from the shock. Up to this time, no signature of vortex pairing appears. In this case we can not invoke the RT instability since at the vortex boundaries the density gradient and the "effective gravity" (associated to the rotational motion of the plasma) are both directed outwards.

In order to identify these instabilities, in Fig. 3 we show the absolute value of the in-plane velocity component (i.e. in the (x,y)-plane) around the top vortex centered at $x \sim 0$, $y \sim 140$. The plasma inside the vortex has been rolled up with a very large velocity in the lateral zone of the vortex (yellow region in Fig. 3). Near this region, but outside the vortex, the magnetosheath plasma goes through a shock which decreases its downstream velocity. Consequently the velocity shear at the vortex boundary increases and considering that the magnetic field is quasi-perpendicular to the $(\mathrm{x}, \mathrm{y})$-plane a secondary $\mathrm{KH}$ instability can develop. We can estimate the growth rate $\gamma_{\mathrm{KH}}$ of this $\mathrm{KH}$ instability as $\gamma_{\mathrm{KH}, \max } \simeq 0.2 V_{\text {shear }} /(2 l)$ (Miura, 1997). Here, $V_{\text {shear }}$ is the 


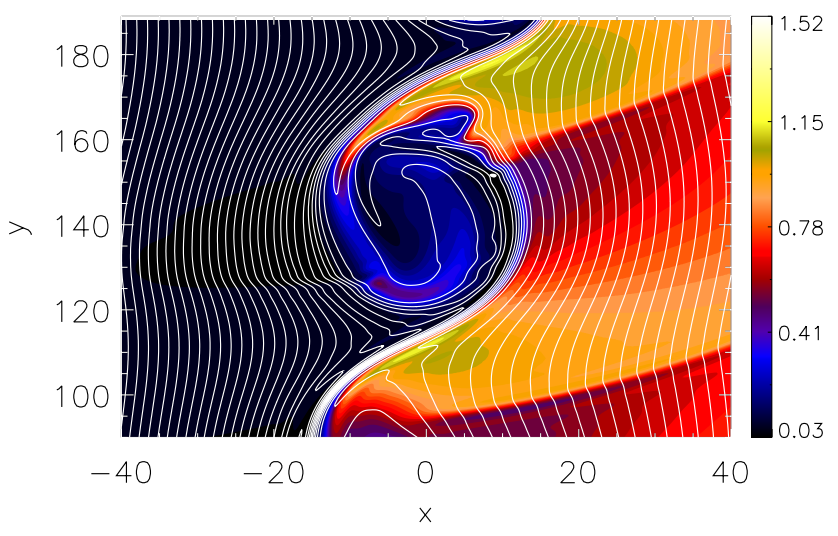

Fig. 2. Shaded density isocontours at $t=156, V_{0}=6$. White curves represent magnetic field lines in the $(\mathrm{x}, \mathrm{y})$-plane.

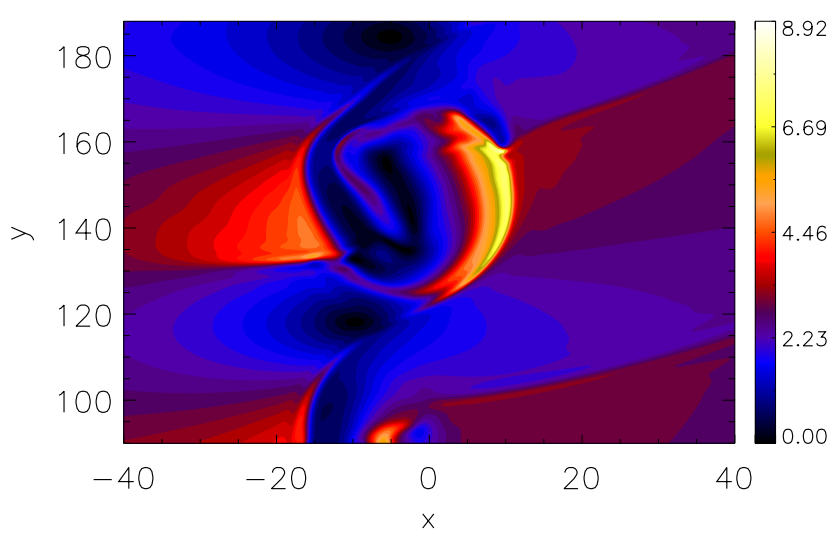

Fig. 3. Shaded isocontours of the absolute value of the velocity component in the (x,y)-plane at $t=160, V_{0}=6$.

jump through the vortex boundary of the velocity tangent to the boundary and $l$ is the thickness of the shear layer. However, even when our simulation is continued for longer times $(t=166)$ we do not observe the development of the KH instability into fully fledged secondary vortex structures. This is due to the fact that the thickness of the shear layer is of the order of the shear length parallel to the flow direction. Thus instabilities with a wavelength larger than the layer thickness, as the fast growing mode, cannot be properly excited.

In Fig. 4 we can observe the same vortex as in Fig. 2 but at $t=160$. We observe that the secondary KH instability starts in correspondence to the shock at the position where it is attached to the vortex. Here the in-plane magnetic field line deformations produced by the perturbed plasma motion allow magnetic reconnection to occur. As a result, in the top arc of the vortex about $y \sim 160$ in Fig. 4 we see the formation of magnetic islands in the magnetic field component in the $(\mathrm{x}, \mathrm{y})$-plane with size of the order of the ion skin depth that are transported by the plasma rotation in its circular type motion.

Increasing the value of $V_{0}$ leads to an increase of the velocity at the vortex boundary in correspondence to the shock

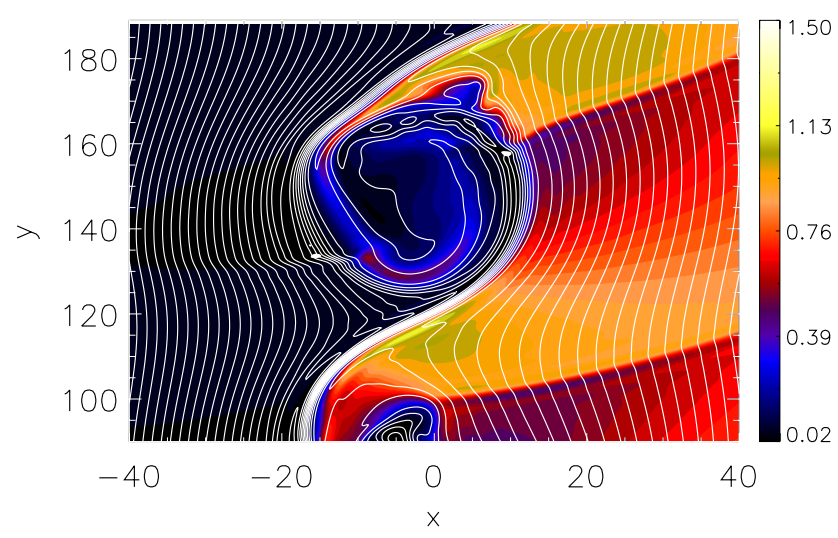

Fig. 4. Shaded density isocontours at $t=160, V_{0}=6$. White curves represent magnetic field lines in the (x,y)-plane.

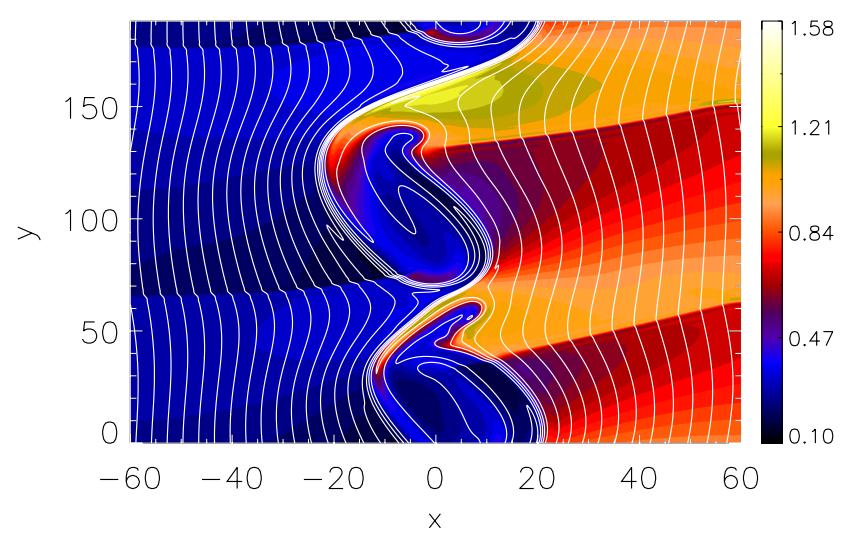

Fig. 5. Shaded density isocontours at $t=330, V_{0}=4.6$. White curves represent magnetic field lines in the $(\mathrm{x}, \mathrm{y})$-plane.

where the secondary $\mathrm{KH}$ instability develops. In this case, taking e.g. $V_{0}=7$, we can observe the detachment of entire vortices that remain inside the magnetosphere (see Fig. 7 in Palermo et al., 2011).

In order to clarify the nature of this secondary instability we performed a different simulation with a smaller density jump corresponding to $\Delta n=0.7$ in Eq. (6). We imposed $\Delta T_{\mathrm{i}}=0.6$ and $\Delta T_{\mathrm{e}}=0.4$ in Eq. (8) and took $T_{0, \mathrm{i}}=0.6$, $T_{0, \mathrm{e}}=0.4$. Since from Eq. (11) we expect a reduced vortex velocity, we imposed a total velocity jump $V_{0}=4.6$ so as to obtain a Mach number $M_{\mathrm{f} R}^{\text {vort }} \simeq 1$, not too different from that of the previous case. In Fig. 5 we observe at $t=330$ the formation of two vortices. We note that the instability growth rate is reduced with respect to the previous case with a larger density jump. Since the wavelength of the "fast growing mode" increases when the density jump $\Delta n$ decreases, the vortices generated by the KH instability are longer in the $\mathrm{y}$ direction (with respect to the shear layer width $L_{\mathrm{eq}}$ that is kept unchanged). As a consequence, a smaller number of vortices are observed in our (fixed size) simulation box. Thus a "weak" density jump between the magnetosheath and the 


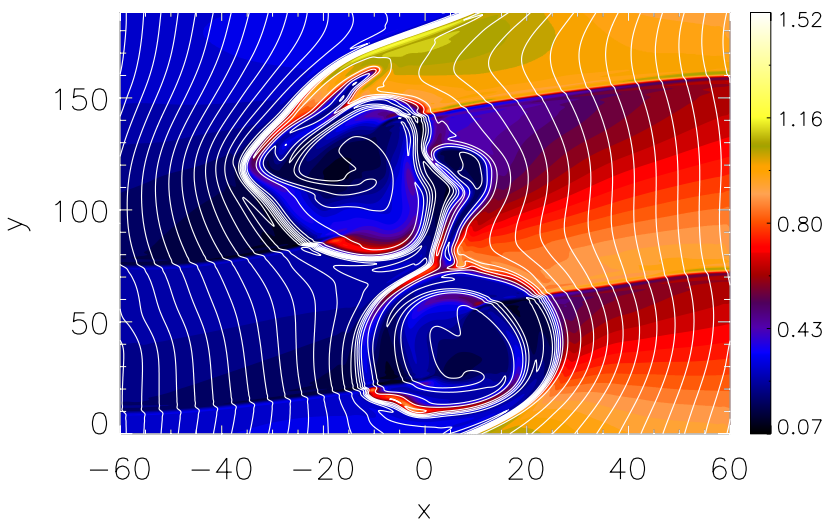

Fig. 6. Shaded density isocontours at $t=350, V_{0}=4$.6. White curves represent magnetic field lines in the (x,y)-plane.

magnetosphere is responsible for the formation, in the early non linear phase of the instability, of vortices with typical size larger than that expected in the case of a strong density jump such as the one observed in typical magnetopause conditions. The structure of these vortices is very similar to those described above for the case with $V_{0}=6$. However conditions in the magnetospheric side are different and we observe the formation of shocks in the magnetosphere in correspondence to each vortex. The main difference with respect to the case with $V_{0}=6$ is that in this $V_{0}=4.6$ case the vortex pairing mechanism can act. This is shown in Fig. 6 where we observe at $t=350$ the early phase of the vortex pairing process between the two vortices. We observe that, as a consequence of the vortex pairing, the vortex at $y \sim 40$ is stretched in the y-direction. This allows for the full development of the secondary KH instability at the boundary of the vortex, as shown in Fig. 7 in a limited region of this vortex $(y \sim 80)$ at $t=370$ by means of the passive tracers. These tracers (see Faganello et al., 2009, for details), purely advected by the velocity field, represent the evolution of the magnetospheric (blue) and magnetosheath (yellow) plasma, separated by the transition region (green and orange).

Finally, we consider an intermediate regime where the flow velocity is reduced to $V_{0}=2$, corresponding to a fast magnetosonic Mach number $M_{\mathrm{f} L / R}^{\text {vort }}=0.62$ then below the super-magnetosonic threshold. We take $\theta=0.05$, and, at variance with magnetosonic conditions, a uniform density $n_{0}$ and temperature profile, $T_{0, \mathrm{i}}=0.5, T_{0, \mathrm{e}}=0.5$ in order to focus on the competition between the vortex pairing and the secondary $\mathrm{KH}$ and reconnection instabilities. The length of the simulation box in the solar wind flow direction is $L_{\mathrm{y}}=30 \pi$, which allows for the formation of two vortices in the central region. This case is similar to the one presented in Faganello et al. (2008c) but with a stronger initial flow. In Fig. 8 we show the passive tracers and the magnetic field lines at $t=275$. An important consequence of the pairing process, is the formation between the vortices of two

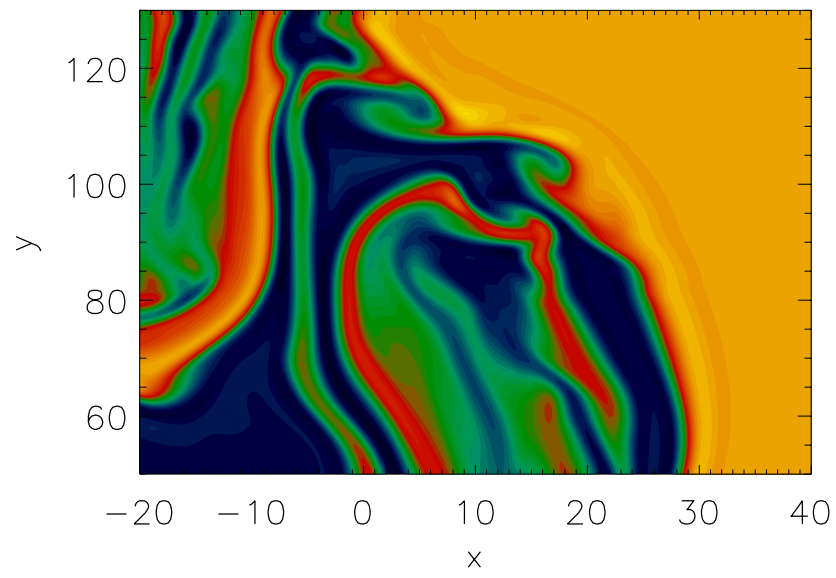

Fig. 7. Passive tracers at $t=370, V_{0}=4.6$. The point where the shock is attached to the vortex is localized at $x=30, y=80$.

strong current sheets (not shown here), driven by the compression associated to the pairing mechanism, similar to what observed in Faganello et al. (2008c).

However, in this case the central large scale ribbon (a region of compressed and nearly parallel magnetic field lines that clearly separate the magnetospheric and the magnetosheath plasmas (Faganello et al., 2008c) is not cut and reconnected, as shown by the evolution of the system in Fig. 9 . Indeed, the presence of a stronger initial velocity field on one side accelerates the pairing process and at the same time drives, in particular near the central reconnection layer, the development of secondary KH instabilities (see Fig. 9) that slow down the reconnection process. In this regime the hydrodynamic interaction between the vortices dominates the non linear dynamics of the system, as can be expected in view of the larger Alfvèn Mach number associated with the in-plane component of the magnetic field (Baty et al., 2003). We recall that this Alfvèn Mach number is a measure of the importance of the magnetic field line tension in the $(\mathrm{x}, \mathrm{y})$ plane.

In Fig. 10 we show the final state of the vortex pairing process from which a single vortex structure has emerged. In this last phase, in the bottom frame, we observe the formation of a pair of shocks marked by the strong density variation (red to yellow) that, starting from the outer regions of the vortex, extend towards the right and the left boundary of the box. The shocks are formed because the vortex rotation and the pairing process lead to a strong modulation of the velocity field at the flank of the vortices. The combined action of the shocks and of the vortex pairing creates a turbulent mixing flow embedding several "magnetic islands" with a typical size of the order of $d_{\mathrm{i}}$, as shown in the top frame of Fig. 10 by the magnetic field lines in the (x,y)-plane. These islands are quite stable and are advected by the rotational flow of the vortex. 


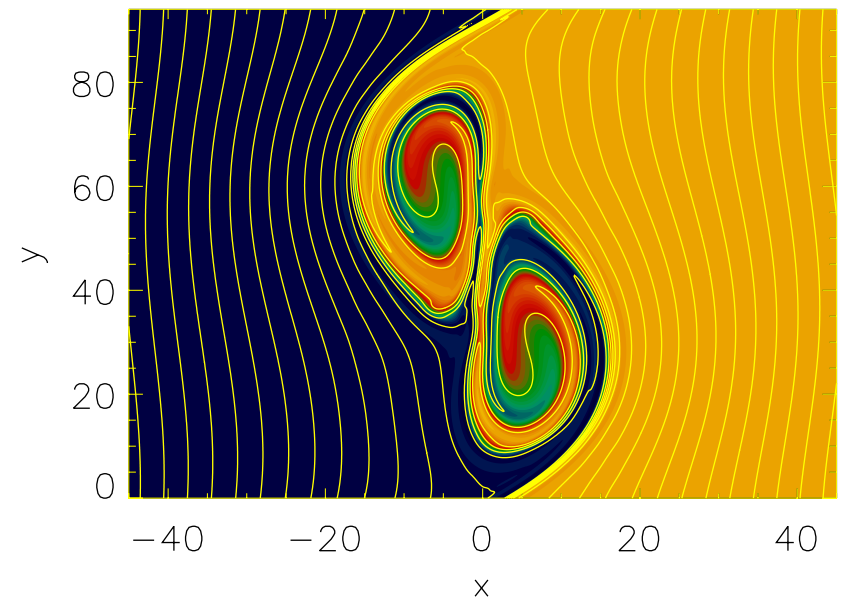

Fig. 8. Shaded isocontours of the passive tracers at $t=275, V_{0}=2$. From the left to the right side along the $\mathrm{x}$-axis, the passive tracers, purely advected by the velocity field, represent the magnetospheric (blue) and magnetosheath (yellow) plasma separated by the transition region (green and orange). Yellow curves represent magnetic field lines in the (x,y)-plane.

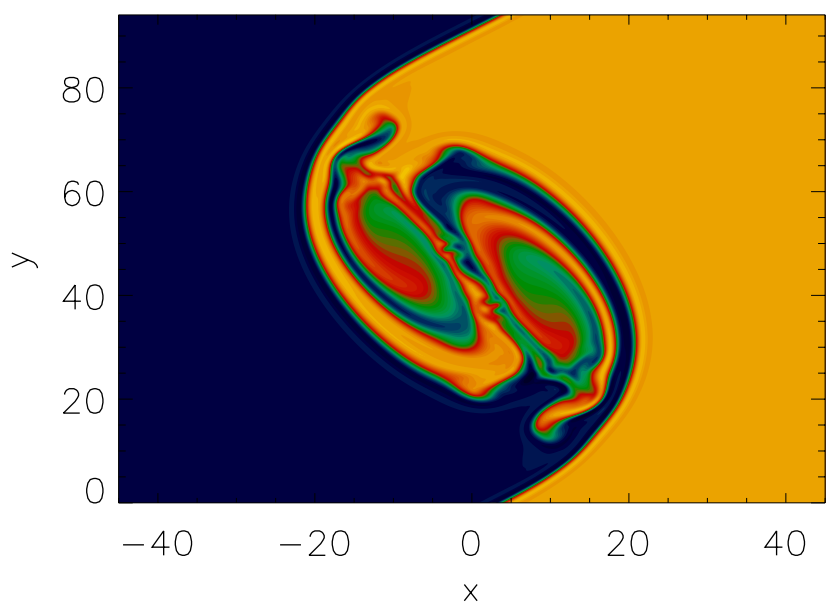

Fig. 9. Shaded isocontours of the passive tracers at $t=300, V_{0}=2$. From the left to the right side along the $\mathrm{x}$-axis, the passive tracers, purely advected by the velocity field, represent the magnetospheric (blue) and magnetosheath (yellow) plasma separated by the transition region (green and orange).

\section{Conclusions}

In this article we have investigated with the help of 2-D twofluid numerical simulations the nonlinear behaviour of the $\mathrm{KH}$ instability in super-magnetosonic regimes of interest for magnetospheric Earth conditions. In particular we have focussed our attention on the role that the shock structures produced by the interaction between the flow and the KH vortices play at the vortex boundaries. These shocks induce a rapid change of the plasma velocity between its upstream and downstream regions which in turns leads to the onset

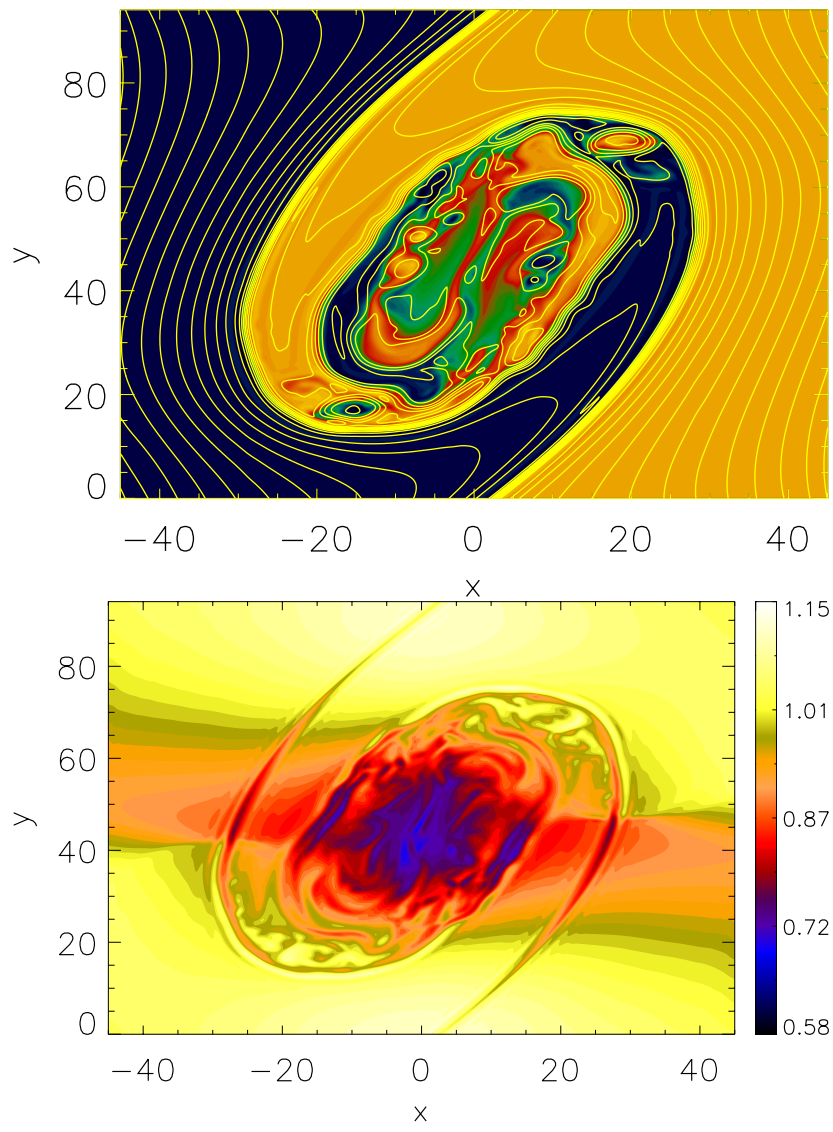

Fig. 10. Shaded isocontours of the passive tracer (top frame) and of the density (bottom frame) at $t=350, V_{0}=2$. From the left to the right side along the $\mathrm{x}$-axis, the passive tracers, purely advected by the velocity field, represent the magnetospheric (blue) and magnetosheath (yellow) plasma separated by the transition region (green and orange). Yellow curves in the top frame represent the magnetic field lines in the $(\mathrm{x}, \mathrm{y})$-plane.

of local secondary instabilities at the vortex boundaries. We have found that these secondary instabilities can develop in super-magnetosonic regimes under a wide range of plasma conditions and we expect that their occurrence is a general feature of the interplay between shocks and KH vortices.

In addition we have investigated the mechanism that makes these instabilities grow and their influence on the dynamical evolution of the vortices. We have identified these instabilities as secondary $\mathrm{KH}$ instabilities that perturb the plasma flow inside the vortices leading to the formation of long lived magnetic islands via magnetic reconnection. In configurations with a large shear velocity field this instability is able to detach the entire vortex structure that afterwards remains completely embedded inside the magnetosphere. As shown in Palermo et al. (2011) these detached vortices consist in large part of magnetosheath plasma decompressed during the vortex generation process at nearly magnetospheric values. Thus this secondary instability represents a new 
efficient channel by which the solar plasma can enter the magnetosphere.

We find that the secondary $\mathrm{KH}$ instability is also present in configurations where the plasma density is uniform where the vortex pairing can act before the shocks can develop. After the shocks have developed, a region of turbulent mixing forms inside the vortices induced by the secondary $\mathrm{KH}$ instability and by the pairing process in correspondence to the shocks.

Finally, even if our 2-D fluid approach should be extended to a 3-D, fully kinetic model (including Earths magnetospheric field lines connection), we may conjecture that the shock structures described in this article should be observed in satellites data as density (and perpendicular magnetic field) transitions connected to vortex structures with a smooth core and fluctuating boundaries due to the onset of secondary instabilities.

Acknowledgements. The research leading to these results has received funding from the European Commission's Seventh Framework Programme (FP7/2007-2013) under the grant agreement SWIFF (project no. 263340, www.swiff.eu). We acknowledge the CINECA Award N. HP10BB99G8, 2011 for the availability of high performance computing resources and support.

Guest Editor M. Balikhin thanks O. A. Pokhotelov and another anonymous referee for their help in evaluating this paper.

\section{References}

Axford, W. I. and Hines, C. O.: A unifying theory of high-latitude. Geophysical phenomena and geomagnetic storms, Can. J. Phys., 39, 1443-1464, 1961.

Baty, H., Keppens, R., and Comte, P.: The two-dimensional magnetohydrodynamic Kelvin-Helmholtz instability: Compressibility and large-scale coalescence effects, Phys. Plasmas, 10, 46614674, 2003.

Belmont, G. and Chanteur, G.: Kelvin-Helmholtz instability: nonlinear evolution, in: Turbulence and Nonlinear Dynamics in MHD Flows, edited by: Meneguzzi, M., Pouquet, A., and Sulem, P., Elsevier Science Publishers, North Holland, 1989.

Chen, S. H., Kivelson, M. G., Gosling, J. T., Walker, R. J., and Lazarus, A. J.: Anomalous aspects of magnetosheath flow and of the shape and oscillations of the magnetopause during an interval of strongly northwards interplanetary magnetic field, J. Geophys. Res., 98, 5727-5742, 1993.

Faganello, M., Califano, F., and Pegoraro, F.: Competing mechanisms of plasma transport in inhomogeneous configurations with velocity shear: the solar-wind interaction with Earth's magnetosphere, Phys. Rev. Lett., 100, 015001, doi:10.1103/PhysRevLett.100.015001, 2008a.

Faganello, M., Califano, F., and Pegoraro, F.: Numerical evidence of undriven, fast reconnection in the solar-wind interaction with Earth's magnetosphere: formation of electromagnetic coherent structures, Phys. Rev. Lett., 101, 105001, doi:10.1103/PhysRevLett.101.105001, 2008b.

Faganello, M., Califano, F., and Pegoraro, F.: Time window for magnetic reconnection in plasma configura- tions with velocity shear, Phys. Rev. Lett., 101, 175003 , doi:10.1103/PhysRevLett.101.175003, 2008c.

Faganello, M., Califano, F., and Pegoraro, F.: Being on time in magnetic reconnection, New J. Phys., 11, 063008, doi:10.1088/13672630/11/6/063008, 2009.

Fairfield, D. H., Otto, A., Mukai, T., Kokubun, S., Lepping, R. P., Steinberg, J. T., Lazarus, A. J., and Yamamoto, T.: Geotail observations of the Kelvin-Helmholtz instability at the equatorial magnetotail boundary for parallel northward fields, J. Geophys. Res., 105, 21159-21173, 2000.

Fujimoto, M., Terasawa, T., Mukai, T., Saito, Y., Yamamoto, T., and Kokubun, S.: Plasma entry from the flanks of the near-Earth magnetotail: Geotail observations, J. Geophys. Res., 103, 43914408, 1998.

Hasegawa, H., Fujimoto, M., Phan, T. D., Réme, H., Balogh, A., Dunlop, M. W., Hashimoto, C., and TanDokoro, R.: Transport of solar wind into Earth's magnetosphere through rolled-up KelvinHelmholtz vortices, Nature, 430, 755-758, 2004.

Kobayashi, Y., Kato, M., Nakamura, K. T. A., Nakamura, T. K. M., and Fujimoto, M.: The structure of Kelvin-Helmholtz vortices with supersonic flow, Adv. Space Res., 41, 1325-1330, 2008.

Lele, S. K.: Compact finite difference schemes with spectral-like resolution, J. Comput. Phys., 103, 16-42, 1992.

Lennartsson, W. and Shelley, E. G.: Survey of 0.1- to $16-\mathrm{keV} / \mathrm{e}$ Plasma Sheet Ion Composition, J. Geophys. Res., 91, 30613076, 1986.

Liu, Z. X. and Hu, Y. D.: Local magnetic reconnection caused by vortices in the flow field, Geophys. Res. Lett., 15, 752-755, 1988.

Matsumoto, Y. and Hoshino, M.: Onset of turbulence induced by a Kelvin-Helmholtz vortex, Geophys. Res. Lett., 31, L02807, doi:10.1029/2003GL018195, 2004.

Mitchell, D. G., Kutchko, F., Williams, D. J., Eastman, T. E., Frank, L. A., and Russell, C. T.: An Extended Study of the LowLatitude Boundary Layer on the Dawn and Dusk Flanks of the Magnetosphere, J. Geophys. Res., 92, 7394-7404, 1987.

Miura, A.: Nonlinear evolution of the magnetohydrodynamic Kelvin-Helmholtz instability, Phys. Rev. Lett., 49, 779-782, 1982.

Miura, A.: Anomalous transport by magnetohydrodynamic KelvinHelmholtz instabilities in the solar wind-magnetosphere interaction, J. Geophys. Res., 89, 801-818, 1984.

Miura, A.: Simulation of Kelvin-Helmholtz Instability at the Magnetospheric Boundary, J. Geophys. Res., 92, 3195-3206, 1987.

Miura, A.: Kelvin-Helmholtz instability for supersonic shear flow at the magnetospheric boundary, Geophys. Res. Lett., 17, 749$752,1990$.

Miura, A.: Kelvin-Helmholtz instability at the magnetospheric boundary: Dependence on the magnetosheath sonic Mach number, J. Geophys. Res., 97, 10655-10675, 1992.

Miura, A.: Compressible magnetohydrodynamic Kelvin-Helmholtz instability with vortex pairing in the two-dimensional transverse configuration, Phys. Plasmas, 4, 2871-2885, 1997.

Nakamura, T. K. M. and Fujimoto, M.: Magnetic reconnection within rolled-up MHD-scale Kelvin-Helmholtz vortices: Twofluid simulations including finite electron inertial effects, Geophys. Res. Lett., 32, L21102, doi:10.1029/2005GL023362, 2005.

Nishida, A., Mukai, T., Yamamoto, T., Saito, Y., and Kokubun, S.: Magnetotail convection in geomagnetically active times, 1 , Dis- 
tance to the neutral lines, J. Geomagn. Geoelectr., 48, 489-501, 1996.

Nykyri, K. and Otto, A.: Plasma transport at the magnetospheric boundary due to reconnection in Kelvin-Helmholtz vortices, Geophys. Res. Lett., 28, 3565-3568, 2001.

Otto, A. and Fairfield, D. H.: Kelvin-Helmholtz instability at the magnetotail boundary: MHD simulation and comparison with Geotail observations, J. Geophys. Res., 105, 21175-21190, 2000.

Palermo, F., Faganello, M., Califano, F., Pegoraro, F., and Le Contel, O.: Compressible Kelvin-Helmholtz instability in super-magnetosonic regimes, J. Geophys. Res., 116, A04223, doi:10.1029/2010JA016400, 2011.

Phan, T. D., Kistler, L. M., Klecker, B., Haerende, G., Paschmann, G., Sonnerup, B. U. Ö, Baumjohann, W., Bavassano-Cattaneo, M. B., Carlson, C. W., DiLellis, A. M., Fornacon, K.-H., Frank, L. A., Fujimoto, M., Georgescu, E., Kokubun, S., Moebius, E., Mukai, T., Øieroset, M., Paterson, W. R., and Reme, H.: Extended magnetic reconnection at the Earth's magnetopause from detection of bidirectional jets, Nature, 404(20), 848-850, 2000.
Spreiter, J. R., Summers, A. L., and Alksne, A. Y.: Hydromagnetic flow around the magnetosphere, Planet. Space Sci., 14, 223-250, 1966.

Tenerani, A., Faganello, M., Califano, F., and Pegoraro, F.: Nonlinear vortex dynamics in an inhomogeneous magnetized plasma with a sheared velocity field, Plasma Phys. Control. Fusion, 53, 015003, doi:10.1088/0741-3335/53/1/015003, 2010.

Valentini, F., Trávníček, P., Califano, F., Hellinger, P., and Mangeney, A.: A hybrid-Vlasov model based on the current advance method for the simulation of collisionless magnetized plasma, J. Comp. Physics, 225, 753-770, 2007. 